\title{
REAL ZEROS OF RANDOM ALGEBRAIC POLYNOMIALS
}

\author{
K. FARAHMAND
}

(Communicated by Lawrence F. Gray)

\begin{abstract}
There are many known asymptotic estimates of the expected number of real zeros of algebraic polynomials with independent random coefficients of equal means. The present paper considers the case when the means of the coefficients are not all necessarily equal. The expected number of crossings of two algebraic polynomials with unequal degree flows from the results.
\end{abstract}

\section{INTRODUCTION}

Let

$$
T(x)=\sum_{i=0}^{n-1} a_{i} x^{i},
$$

where $a_{0}, a_{1}, \ldots, a_{n-1}$ is a sequence of independent, normally distributed random variables with variances one; let $N(\alpha, \beta)$ be the number of real zeros of $T(x)$ in the interval $(\alpha, \beta)$, where multiple roots are counted only once. Kac [6] has considered the case when the means of the $a_{i}$ 's $(i=0,1, \ldots, n-1)$ are all zero and found that $E N(-\infty, \infty)$, the mathematical expectation of $N(-\infty, \infty)$, is asymptotic to $(2 / \pi) \log n$. Later Ibragimov and Maslova [4] obtained the same asymptotic number of real roots for a case that includes the results due to Kac [6, 7], Littlewood and Offord [8], and others. They consider the case when the coefficients $a_{i}$ belong to the domain of attraction of normal laws with zero mean. Their method allowed them [5] to show that if the means of all the coefficients are equal but nonzero, then the expected number of real roots asymptotically reduces by half. This remarkable reduction of the real roots by half persists in the work of Farahmand $[2,3]$ when he studied the real roots of the equation $T(x)=K$ for any nonzero $K$.

Here we consider the case when the means of the coefficients are not all necessarily equal. The theorems show that in the interval $(-1,1)$, only the means of the first $n^{\prime}$ coefficients, $n^{\prime}=[n / k]$, where $k \geq 1$ is a constant, have a decisive affect on the behavior of the polynomial, and only if these means are

Received by the editors April 16, 1990 and, in revised form, September 20, 1990.

1980 Mathematics Subject Classification (1985 Revision). Primary 60G99; Secondary 42BXX.

Key words and phrases. Number of real roots, number of crossings, Kac-Rice formula, random algebraic polynomial. 
zero, nonzero bounded, or nonzero unbounded are different results obtained for $E N(-1,1)$. While outside this interval the mean of the last $n^{\prime}$ term dictates the behavior of the polynomial. These results, of interest in their own right, are important as they lead to the expected number of crossings of two polynomials with different degrees. The set of equations

$$
y=F(x)=\sum_{i=0}^{n-1} b_{i} x^{i} \text { and } y=Q(x)=\sum_{j=0}^{n^{\prime}-1} c_{j} x^{j}
$$

represent two families of curves in the $x y$-plane. Then

$$
F(x)-Q(x)=\sum_{i=0}^{n^{\prime}-1}\left(b_{i}-c_{i}\right) x^{i}+\sum_{i=n^{\prime}}^{n-1} b_{i} x^{i}
$$

can be represented as $\sum_{i=0}^{n-1} a_{i} x^{i}$ where $a_{i}=\left(b_{i}-c_{i}\right)$ for $0 \leq i \leq n^{\prime}-1$ and $a_{i}=b_{i}$ for $n^{\prime} \leq i \leq n-1$, which is, indeed, in the form of $T(x)$. Hence $E N(-\infty, \infty)$ would be the expected number of crossings of $F(x)$ with $Q(x)$ and consequently is only governed by the difference between means of $b_{i}$ and $c_{i}$ for $0 \leq i \leq n^{\prime}$ in the interval $(-1,1)$, and by the means of $b_{i}$ for $n^{\prime}<i \leq n-1$ outside this interval. The results are stated in the following:

Theorem 1. If the coefficients $a_{i}, i=0,1, \ldots, n-1$, of $T(x)$ are normally distributed with variances one and means $\mu_{1}$ and $\mu_{2}$ for $0 \leq i \leq n^{\prime}-1$ and $n^{\prime} \leq i \leq n-1$, respectively, where $n^{\prime}=n / k$ for $k>1$ any constant such that $k=o(\sqrt{\log n})$, then the expected number of real roots of $T(x)=0$ satisfies

$$
E N(-1,1) \sim(1 / 2 \pi) \log n \text { if } \mu_{1} \neq 0 \text { bounded and } \mu_{2}=o\left(n^{\gamma}\right),
$$

and

$E N(-\infty,-1)+E N(1, \infty) \sim(1 / 2 \pi) \log n$ if $\mu_{2} \neq 0$ bounded and $\mu_{1}=o\left(n^{\gamma}\right)$, where $\gamma$ is any positive constant.

Theorem 2. If the coefficients $a_{i}$ are distributed according to the assumption of Theorem 1, then for $k^{1 / 2}=o\left(\sqrt{\log n}-\log \mu_{1}^{2} / \sqrt{\log n}\right)$,

$$
E N(-1,1) \sim(1 / 2 \pi) \log \left(n / \mu_{1}^{2}\right)
$$

if $\mu_{1} \rightarrow \infty$ as $n \rightarrow \infty$ such that $\mu_{1}=o(\sqrt{n})$ and $\mu_{2}=o\left(n^{\gamma}\right)$ and

$$
E N(-\infty,-1)+E N(1, \infty) \sim(1 / 2 \pi) \log \left(n / \mu_{2}^{2}\right)
$$

if $\mu_{2} \rightarrow \infty$ as $n \rightarrow \infty$ such that $\mu_{2}=o(\sqrt{n})$ and $\mu_{1}=o\left(n^{\gamma}\right)$, where $\gamma$ is any positive constant.

Theorem 3. Under the assumption of Theorem 1 , for $k=o(\sqrt{\log n})$,

$$
E N(-1,1) \sim(1 / \pi) \log n \quad \text { if } \mu_{1}=0 \text { and } \mu_{2}=o(\sqrt{n})
$$


and

$$
E N(-\infty,-1)+E N(1, \infty) \sim(1 / \pi) \log n \text { if } \mu_{2}=0 \text { and } \mu_{1}=o(\sqrt{n}) .
$$

Corollary. If the coefficients $b_{i}$ and $c_{j}$ of $F(x)$ and $Q(x)$ are normally distributed with means $\mu_{2}$ and $\mu_{1}^{\prime}$, then the expected number of crossings of these two polynomials comply with Theorem 1 to 3 with $\mu_{1}=\mu_{2}-\mu_{1}^{\prime}$.

\section{A FORMULA FOR THE EXPECTED NUMBER OF REAL ROOTS}

First we obtain a formula for the average number of real roots of the equation $T(x)=0$ under the assumptions of Theorem 1 or 2 . Indeed, the result would remain valid for Theorem 3 by letting $\mu_{1}=0$. Let

$$
\Phi(t)=(2 \pi)^{-1 / 2} \int_{-\infty}^{t} \exp \left(-y^{2} / 2\right) d y
$$

and

$$
\phi(t)=\Phi^{\prime}(t)=(2 \pi)^{-1 / 2} \exp \left(-t^{2} / 2\right) .
$$

Then by using the expected number of level crossings developed by Cramer and Leadbetter [1, p. 285], we obtain

$$
E N(\alpha, \beta)=\int_{\alpha}^{\beta}(B / A)\left(1-\theta^{2}\right)^{1 / 2} \phi\left(\lambda_{1} / A\right)[2 \phi(\eta)+\eta\{2 \Phi(\eta)-1\}] d x
$$

where

$$
\begin{gathered}
A^{2}=\operatorname{Var}\{T(x)\}, \quad B^{2}=\operatorname{Var}\left\{T^{\prime}(x)\right\}, \\
\theta=(A B)^{-1} \operatorname{Cov}\left\{T(x), T^{\prime}(x)\right\}, \quad \lambda_{1}=E\{T(x)\}, \\
\lambda_{2}=E\left\{T^{\prime}(x)\right\}, \quad \text { and } \eta=\left(1-\theta^{2}\right)^{-1 / 2}\left(\lambda_{2}-B \theta \lambda_{1} / A\right) / B .
\end{gathered}
$$

Since the coefficients of $T(x)$ are independent normally distributed random variables, we can easily show that

$$
\begin{gathered}
\lambda_{1}=\mu_{1} \sum_{i=0}^{n^{\prime}-1} x^{i}+\mu_{2} \sum_{i=n^{\prime}}^{n-1} x^{i}, \quad \lambda_{2}=\mu_{1} \sum_{i=0}^{n^{\prime}-1} i x^{i-1}+\mu_{2} \sum_{i=n^{\prime}}^{n-1} i x^{i-1}, \\
A^{2}=\sum_{i=0}^{n-1} x^{2 i}, \quad B^{2}=\sum_{i=0}^{n-1} i^{2} x^{2 i-2}, \\
C=\operatorname{Cov}\left\{T(x), T^{\prime}(x)\right\}=\sum_{i=0}^{n-1} i x^{2 i-1}, \quad \eta=\left(A^{2} \lambda_{2}-C \lambda_{1}\right) / A \sqrt{A^{2} B^{2}-C^{2}}
\end{gathered}
$$


Hence, from (2.1) and since $\Phi(t)=1 / 2+(\pi)^{-1 / 2} \operatorname{erf}(t / \sqrt{2})$, we have the extension of the Kac-Rice formula [9]

$$
\begin{aligned}
E N(\alpha, \beta)= & \int_{\alpha}^{\beta}\left[\left(\Delta / \pi A^{2}\right) \exp \left\{-\left(A^{2} \lambda_{2}^{2}+B^{2} \lambda_{1}^{2}-2 C \lambda_{1} \lambda_{2}\right) / 2 \Delta^{2}\right\} d x\right. \\
& +\int_{\alpha}^{\beta}(\sqrt{2} / \pi)\left|A^{2} \lambda_{2}-C \lambda_{1}\right| A^{-3} \exp \left(-\lambda_{1}^{2} / 2 A^{2}\right) \\
\left.\quad \times \operatorname{erf}\left(\left|A^{2} \lambda_{2}-C \lambda_{1}\right| / A \Delta \sqrt{2}\right)\right] d x &
\end{aligned}
$$

say, where $\Delta^{2}=A^{2} B^{2}-C^{2}$.

\section{Proof of Theorem 1}

Observe that by virtue of the relation

$$
T(x)=x^{n-1} \sum_{i=0}^{n-1} a_{n-i-1} x^{-i}=x^{n-1} T^{*}(y), \quad y=1 / x,
$$

there corresponds to each zero of the polynomial $T(x)$ in the interval $(0,1)$ (or $(-1,0)$ ) a zero of the polynomial $T^{*}(x)$ on the half-line $(1, \infty)$ (or $(-\infty,-1))$. Let us interchange $\mu_{1}$ and $\mu_{2}$ and replace $k$ by $k /(k-1)$ in $T^{*}(x)$; and denote by $N^{*}$ the number of real roots of such a polynomial. Since the distribution of the latest polynomial is identical to that of $T(x)$, we have $E N(0,1)=E N^{*}(1, \infty)$ and $E N(-1,0)=E N^{*}(-\infty,-1)$. Therefore it suffices to determine the asymptotic behavior of $\operatorname{EN}(-1,0)$ and $\operatorname{EN}(0,1)$ and use them to establish the values of $E N(-\infty,-1)$ and $E N(1, \infty)$. In doing so we first consider the interval $(0,1)$. Let $0 \leq x \leq 1-n^{\varepsilon-1}$, where $\varepsilon=\left\{\log \left(\log n^{\gamma}\right) / \log n, r=\gamma^{\prime} k\right.$, and $\gamma^{\prime}=\max \left\{\gamma^{2}, 10\right\}$. Then, since $x^{n}<$ $x^{n^{\prime}} \leq \exp \left(-n^{\prime} n^{\varepsilon-1}\right)=n^{-n^{\prime} r^{\prime} n}=n^{-\gamma^{\prime}}$, we can show

$$
\begin{gathered}
\lambda_{1}=\mu_{1}\left(1-x^{n^{\prime}}\right) /(1-x)+\mu_{2}\left(x^{n^{\prime}}-x^{n}\right) /(1-x) \\
=\mu_{1} /(1-x)+o\left\{\left(\mu_{1}+\mu_{2}\right) / k n^{\gamma^{\prime}-1} \log n\right\} ; \\
\lambda_{2}=\mu_{1}\left\{\left(1-x^{n^{\prime}}\right) /(1-x)^{2}-n^{\prime} x^{n^{\prime}-1} /(1-x)\right\} \\
+\mu_{2}\left\{x^{n^{\prime}}-x^{n}-(1-x)\left(n^{\prime} x^{n^{\prime}-1}-n x^{n-1}\right)\right\} /(1-x)^{2} \\
=\mu_{1} /(1-x)^{2}+o\left\{\left(\mu_{1}+\mu_{2}\right) / k n^{\gamma^{\prime}-2} \log n\right\} ; \\
A^{2}=\left(1-x^{2 n}\right) /\left(1-x^{2}\right)=1 /\left(1-x^{2}\right)+o\left(1 / k n^{2 \gamma^{\prime}-1} \log n\right) ; \\
B^{2}=\left(-x^{2 n+2}-x^{2 n}+x^{2}+1-n^{2} x^{2 n+2}+2 n^{2} x^{2 n}\right. \\
\left.\quad-n^{2} x^{2 n-2}+2 n x^{2 n+2}+2 n x^{2 n}\right)\left(1-x^{2}\right)^{-3} \\
=\left(1+x^{2}\right) /\left(1-x^{2}\right)^{3}+o\left(1 / k^{2} n^{2 \gamma^{\prime}-5} \log n\right) ;
\end{gathered}
$$




$$
\begin{aligned}
C & =x\left(1-x^{2 n}\right) /\left(1-x^{2}\right)^{2}-n x^{2 n-1} /\left(1-x^{2}\right) \\
& =x /\left(1-x^{2}\right)^{2}+o\left(1 / k^{2} n^{2 y^{\prime}-2} \log ^{2} n\right) .
\end{aligned}
$$

Hence from (3.3)-(3.5), we can write

$$
\Delta^{2}=1 /\left(1-x^{2}\right)^{4}+o\left(1 / k^{3} n^{2 \gamma^{\prime}-6} \log ^{2} n\right),
$$

which together with (3.1)-(3.5), give

$$
\begin{aligned}
& \left(A^{2} \lambda_{2}^{2}+B^{2} \lambda_{1}^{2}-2 C \lambda_{1} \lambda_{2}\right) / 2 \Delta^{2} \\
& \quad=\mu_{1}^{2}(1+x) /(1-x)+o\left\{k^{2}\left(\mu_{1}+\mu_{2}\right)^{2} \log ^{2} n / n^{\gamma^{\prime}+1}\right\}
\end{aligned}
$$

and

$$
\Delta / A^{2}=1 /\left(1-x^{2}\right)+o\left(k / n^{2 \gamma^{\prime}-2}\right) .
$$

Therefore, using $\exp (u)=1+o(u)$ for all sufficiently small $u$, from (3.7) and (3.8) and since $\gamma^{\prime} \geq 10$, we can obtain

$$
\begin{aligned}
I_{1}\left(0,1-n^{\varepsilon-1}\right) & =(1 / \pi) \int_{0}^{1-n^{\varepsilon-1}}\left[\left(1-x^{2}\right)^{-1} \exp \left\{-\mu_{1}^{2}(1+x) /(1-x)\right\}\right. \\
& \left.+o\left\{\left(\mu_{1}+\mu_{2}\right)^{2} n^{-4}\right\}\right] d x \\
& \leq(1 / \pi) \int_{0}^{1-n^{\varepsilon-1}}(1-x)^{2} \exp \left\{-2 \mu_{1}^{2} /(1-x)\right\} d x \\
& \leq\left(1 / 2 \pi \mu_{1}^{2}\right) \exp \left(-2 \mu_{1}^{2}\right) .
\end{aligned}
$$

It so happens that for the range $1-n^{\varepsilon-1}<x \leq 1$ we cannot find out the dominant terms for (3.1)-(3.5). But since

$$
\Delta / A^{2}<(2 n-1)^{1 / 2}(1-x)^{-1 / 2}
$$

for all sufficiently large $n$, we can write

$$
I_{1}\left(1-n^{\varepsilon-1}, 1\right)<\int_{1-n^{\varepsilon-1}}^{1}\left(\Delta / \pi A^{2}\right) d x=o(\sqrt{k \log n}) .
$$

To evaluate $I_{2}(0,1)$, we notice that since $\frac{d}{d x}\left(\lambda_{1} / A\right)=A^{-3}\left(\lambda_{2} A^{2}-\lambda_{1} C\right)$, we have

$$
I_{2}(0,1) \leq(2 \pi)^{-1 / 2} \int_{\mu_{1}}^{\xi} \exp \left(-u^{2} / 2\right) d u \leq \frac{1}{2}
$$

where $u=\lambda_{1} / A$ and $\xi=\left\{\mu_{1} n^{\prime}+\mu_{2}\left(n-n^{\prime}\right)\right\} / n$. Hence from (3.9), (3.11), and (3.12), we obtain

$$
E N(0,1)=o(\sqrt{k \log n}) .
$$


In the following we find $E N(-1,0)$. Let $y=-x$, then by using (3.7) and (3.8) and since $\gamma^{\prime} \geq 10$, we can obtain

$$
\begin{array}{r}
I_{1}\left(-1+n^{\varepsilon-1}, 0\right)=\int_{0}^{1-n^{\varepsilon-1}}\left[\left\{1 / \pi\left(1-y^{2}\right)\right\} \exp \left\{-\mu_{1}^{2}\left(1-y^{2}\right) /\left(1+y^{2}\right)\right\}\right. \\
\left.+o\left\{\left(\mu_{1}+\mu_{2}\right) n^{-6}\right\}\right] d y .
\end{array}
$$

From (3.14) and since $\mu_{1}$ is bounded and $\varepsilon \rightarrow 0$ as $n \rightarrow \infty$, we have

$$
\begin{aligned}
I_{1}\left(-1+n^{\varepsilon-1}, 0\right) & \leq(1 / \pi) \int_{0}^{1-n^{\varepsilon-1}} 1 /\left(1-y^{2}\right) d y \\
& =(1 / 2 \pi)\left\{(1-\varepsilon) \log n+\log \left(2-n^{\varepsilon-1}\right)\right\} \\
& <(1 / 2 \pi) \log n+0.2 .
\end{aligned}
$$

Hence from (3.15) and by noticing that (3.11) would remain valid for $x \in(-1$, $\left.-1+n^{\varepsilon-1}\right)$, we obtain

$$
E N(-1,0) \leq(1 / 2 \pi) \log n+o(\sqrt{k \log n})
$$

In order to obtain a lower estimate for $E N(-1,0)$ from (3.14) for $\lambda=2 \mu_{1}$ and $t=\lambda(1-y)$ and all sufficiently large $n$, we can write

$$
\begin{aligned}
E N(-1,0) & >I_{1}\left(-1+n^{\varepsilon-1},-1\right) \\
& >\int_{0}^{1-n^{\varepsilon-1}}\left\{1 / \pi\left(1-y^{2}\right)\right\} \exp \left\{-2 \mu_{1}^{2}\left(1-y^{2}\right) /(1+y)^{2}\right\} d y \\
& >(1 / 2 \pi) \int_{\lambda n^{\varepsilon-1}}^{\lambda}(1 / t) \exp (-t) d t \\
& =\{(1-\varepsilon) / 2 \pi\} \log n-(1 / 2 \pi) \int_{\lambda n^{\varepsilon-1}}^{\lambda}\{1-\exp (-t)\} / t d t \\
& =(1 / 2 \pi) \log n-(1 / 2 \pi) \int_{0}^{\lambda}\{1-\exp (-t)\} / t d t+o\left(\lambda n^{\varepsilon-1}\right) \\
& =(1 / 2 \pi) \log n+o(\lambda)
\end{aligned}
$$

since $(\lambda / n) \rightarrow 0$ as $n \rightarrow \infty$. Finally noticing that (3.12) remains valid for $-1 \leq x \leq 0$, it together with (3.13), (3.16), and (3.17) gives the asymptotic formula

$$
E N(-1,1) \sim(1 / 2 \pi) \log n,
$$

which completes the proof of Theorem 1 .

\section{Proof of Theorem 2}

If the assumption of $\mu_{1}$ being bounded is relaxed and allowed to tend to infinity as $n \rightarrow \infty$ such that $\mu_{1}=o(\sqrt{n})$, the inequalities in (3.9), (3.11), (3.12), and hence (3.13) remain valid. In order to evaluate $E(-1,0)$ let $\lambda=$ 
$\mu_{1}^{2} / 4$. Then from (3.14) and for all sufficiently large $n$, we can write

$$
\begin{aligned}
I_{1}\left(-1+n^{\varepsilon-1}, 0\right) \leq & (1 / \pi) \int_{0}^{1-n^{\varepsilon-1}}\left\{1 /\left(1-y^{2}\right)\right\} \exp \left\{-\lambda\left(1-y^{2}\right)\right\} d y \\
< & (1 / \pi) \int_{0}^{1-n^{\varepsilon-1}}\left(1-y^{2}\right)^{-1}\left\{1-\lambda\left(1-y^{2}\right)\right\} d y \\
< & (1 / \pi) \int_{0}^{1-n^{\varepsilon-1}}\left[\left(1-y^{2}\right)^{-1}-\lambda /\left\{1+\lambda\left(1-y^{2}\right)\right\}\right] d y \\
< & \{(1-\varepsilon) / 2 \pi\}\left[\log n+\log \left(2-n^{\varepsilon-1}\right)\right] \\
& -(1 / 2 \pi)(1+1 / \lambda)^{-1 / 2} \log \left\{\frac{(1+1 / \lambda)^{1 / 2}+1-n^{\varepsilon-1}}{(1+1 / \lambda)^{1 / 2}-1+n^{\varepsilon-1}}\right\} \\
\leq & (1 / 2 \pi) \log \left(n / \mu_{1}^{2}\right)+0.3 .
\end{aligned}
$$

Hence from (3.11), (3.12), and (4.1), we have

$$
E N(-1,0) \leq(1 / 2 \pi) \log \left(n / \mu_{1}^{2}\right)+o(\sqrt{k \log n}) .
$$

To find a lower estimate of $E N(-1,0)$ from (3.17) we have

$$
\begin{aligned}
E N(-1,0) \geq & \{(1-\varepsilon) / 2 \pi\} \log n-(1 / 2 \pi) \int_{0}^{\lambda}\{1-\exp (-t)\} / t d t \\
& +(1 / 2 \pi) \int_{0}^{\lambda n^{\varepsilon-1}}\{1-\exp (-t)\} / t d t .
\end{aligned}
$$

Since $\left(\lambda n^{\varepsilon-1}\right) \rightarrow 0$ as $n \rightarrow \infty$, it follows that the last integral is $\left(\lambda n^{\varepsilon-1}\right)+$ $o\left(\lambda^{2} n^{2 \varepsilon-2}\right)$. Also

$$
\begin{aligned}
\int_{0}^{\lambda}\{1-\exp (-t)\} / t d t & =\int_{0}^{1}\{1-\exp (-t)\} / t d t+\int_{1}^{\lambda}\{1-\exp (-t)\} / t d t \\
& <\log \lambda+1
\end{aligned}
$$

for all sufficiently large $n$. Then from (4.3) and (4.4) we have

$$
E N(-1,0) \geq(1 / 2 \pi) \log \left(n / \mu_{1}^{2}\right)-0.53,
$$

which together with (4.1) completes the proof of Theorem 2 .

\section{Proof of Theorem 3}

For $\mu_{1}=0$ from (3.8) we obtain

$$
\begin{aligned}
I_{1}\left(0,1-n^{\varepsilon-1}\right) & =I_{1}\left(-1+n^{\varepsilon-1}, 0\right) \\
& =(1 / \pi) \int_{0}^{1-n^{\varepsilon-1}}\left(1-x^{2}\right)^{-1} d x+o(1 / n) \\
& \sim(1 / 2 \pi) \log n .
\end{aligned}
$$


Hence from $(3.11),(3.12),(3.16),(3.17)$, and $(5.1)$, we have the proof of Theorem 3 .

\section{ACKNOWLEDGMENTS}

The author would like to thank the referee for his constructive suggestions, which improved the earlier version of this paper. The final revision of this paper has been prepared while the author was visiting the Department of Mathematics at University of Bristol. This research was supported by the Foundation for Research and Development (FRD) of South Africa.

\section{REFERENCES}

1. H. Cramer and M. R. Leadbetter, Stationary and related stochastic process, Wiley, New York, 1967.

2. K. Farahmand, On the average number of real roots of a random equation, Ann. Probab. 14 (1986), 702-709.

3. _ The average number of level crossings of a random algebraic polynomial, Stochastic Anal. Appl. 6 (1988), 247-272.

4. I. A. Ibragimov and N. B. Maslova, On the expected number of real zeros of random polynomials. I. Coefficients with zero means, Theory Probab. Appl. 16 (1971), 228-248.

5. _ On the expected number of real zeros of random polynomials. II. Coefficients with non-zero means, Theory Probab. Appl. 16 (1971), 485-493.

6. M. Kac, On the average number of real roots of a random algebraic equation, Bull. Amer. Math. Soc. 49 (1943), 314-320.

7. _ - On the average number of real roots of a random algebraic equation, Proc. London Math. Soc. 50 (1949), 390-408.

8. J. E. Littlewood and A. C. Offord, On the number of real roots of a random algebraic equation, Proc. Cambridge Philos. Soc. 35 (1939), 133-148.

9. S. O. Rice, Mathematical theory of random noise, Bull. System Tech. J. 25 (1945), 46-156.

DepartMent of Mathematics, University of Ulster, Jordanstown, Co. ANTRim BT37 OQB, UNITED KINGDOM 